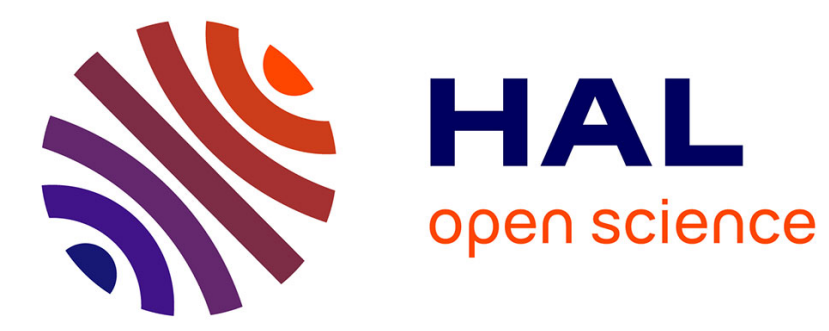

\title{
Some remarks on representations of jet groups and gauge groups
}

Sergio Albeverio, Bruno Torrésani

\section{To cite this version:}

Sergio Albeverio, Bruno Torrésani. Some remarks on representations of jet groups and gauge groups. Journal of Mathematical Physics, 1994, 35 (9), pp.4897-4908. 10.1063/1.530821 . hal-01305436

\section{HAL Id: hal-01305436 \\ https://hal.science/hal-01305436}

Submitted on 21 Mar 2018

HAL is a multi-disciplinary open access archive for the deposit and dissemination of scientific research documents, whether they are published or not. The documents may come from teaching and research institutions in France or abroad, or from public or private research centers.
L'archive ouverte pluridisciplinaire HAL, est destinée au dépôt et à la diffusion de documents scientifiques de niveau recherche, publiés ou non, émanant des établissements d'enseignement et de recherche français ou étrangers, des laboratoires publics ou privés. 


\title{
SOME REMARKS ON REPRESENTATIONS OF JET GROUPS AND GAUGE GROUPS
}

\author{
S. Albeverio*, B.Torresani ${ }^{* *}$
}

\begin{abstract}
We study irreducible unitary representations of the three first jet (or Leibniz) extensions $G_{m}^{k}$ of a compact group $G$, focusing on the neighborhood of the trivial representation.

We also describe some associated irreducible unitary (continuous tensor product) representations of the gauge (or current) groups of mappings from a Riemannian manifold into $G_{m}^{k}$.
\end{abstract}

1992

CPT-92/P.2709

* : Fakultät für Mathematik, Ruhr - Universität,

D-4630 Bochum 1 (Germany) ;

SFB - 237 - Essen - Bochum - Düsseldorf ;

Bibos - Bielefeld ;

CERFIM - Locarno (Switzerland).

** : Research fellowship of the Alexander Von Humboldt - Stiftung. 


\section{I - Introduction}

Since the early seventies, a great attention has been paid to the representation theory of a special class of infinite-dimensional Lie groups, called gauge groups, i.e. groups of the form Map $(M, G), M$ being a Riemannian manifold and $G$ a locally compact Lie group. Here, "Map" stands for any functional setting of interest, but we will restrict ourselves throughout this paper to the groups $G^{M}=C_{0}^{k}(M, G)$ of smooth compactly supported mappings, for the sake of simplicity.

One of the main motivations for the study of these groups is that they play an important role in the context of gauge theories, and the understanding of their representation theory would make easier the construction of quantum theories. Another main motivation was the generalisation of distribution theory to a non-commutative setting. Indeed, I.M. Gelfand and his collaborators remarked that irreducible unitary representations of $G^{M}$ give natural non-commutative generalisations of the unitary characters of $\mathbf{C}^{M}$, that are nothing but complex exponentials of distributions on $M$. Finally, the discovery of affine Kac-Moody algebras and groups (loop groups), special cases of gauge algebras and groups for which $M=T^{1}$, has generated an important activity in the field. There is now a quite well understood highest weight representation theory for affine Kac-Moody algebras, that integrates to a unitary representation theory for the corresponding Kac-Moody groups [1] $[2]$.

Unfortunately, such algebraic techniques fail to generalize to higher-dimensional $M$ manifolds [3], [4], except in some particular cases [2], [5], [6].

In this paper, we will be concerned with the non-commutative distributions point of view (see e.g. [7], [8], [9], [10], [11]; see also [12], [13] for topological background). In [14], the authors constructed an irreducible unitary representation of $S L(2, \mathbf{R})^{M}$, realised in six different ways. It was in particular realised as a continuous tensor product [15], [16],[17], and generalised later to any locally compact group with a non-trivial representation-valued first cohomology group (notice that such an assumption excludes all the simple groups, except $S O_{0}(n, 1)$ and $\left.S U(n, 1)\right)$. The construction was modified later, to apply to compact groups, yielding the so-called energy representations [18] [19] [20]. Such representations have been widely discussed in the literature (see [7] and references therein). They are essentially based on an extension of the compact group by a nilpotent group, yielding the first order Leibniz group of $G$, to which the previous construction can be applied. The use of Leibniz groups has also been proposed in [17]. We study here the three first 
Leibniz extensions of a compact group $G$, to investigate the possibility of constructing associated continuous tensor product representations of $G^{M}$. Using Mackey's induced representations theory, we classify their irreducible unitary representations, and study the neighborhood of the trivial representation (which is closely connected to the representationvalued cohomology groups of order 1).

In particular, we show that the Leibniz extensions of order $2, G_{m}^{2}$, do not yield non trivial continuous tensor product representations of $G^{M}$. In the case of the Leibniz exten-

sions of order $3, G_{m}^{3}$, we show that the trivial representation is not isolated in $\widehat{G_{m}^{3}}$ (where $\widehat{G}$ stands for the unitary dual of $G$ ). Unfortunately, we do not have for the moment any example of associated representation of $G^{M}$. Finally, returning to the case of the first order Leibniz extension, we describe some associated energy representations.

\section{Dedication :}

This paper was written after the untimely departure of our dearest friend Raphael Høegh-Krohn, early 88. It is based on discussions we had with him in Oslo, Bochum and Marseille, in the two last years of his life as part of a general project on the study of non-commutative distributions, in which Raphael had been a most inspiring force.

In fact, in the last years of his life he was deeply concerned with noncommutative distributions which he considered as basic structures of universal relevance, natural infinitedimensional extensions of Lie groups. The present study, to which he also provided the basic ideas, was for him just a component in a greater design. It was extremely sad to carry through this work without him and we missed very much his deep insight.

We dedicate this paper to his beloved memory.

\section{II - Non-commutative distributions and the Vershik-Gel- fand-Graev construction :}

Let us start with a short description of the basic concepts of representations of gauge groups. Let $G$ be a locally compact Lie group, and let $M$ be a Riemannian manifold. Consider the group (gauge group)

$$
G^{M}=\mathcal{D}(M, G)=C_{0}^{\infty}(M, G)
$$

with pointwise group multiplication : 


$$
(\varphi \cdot \psi)(x)=\varphi(x) \psi(x) \quad \varphi, \psi \in G^{M}, x \in M
$$

Such an infinite-dimensional group can be provided with a Schwartz topology (see [7]). We will be interested in (strongly) continuous unitary representations

$$
\mathcal{U}: G^{M} \rightarrow G L(\mathcal{H})
$$

of $G^{M}$ in a Hilbert space $\mathcal{H}$, which provide non-commutative distributions on $M$, as explained in the introduction.

The simplest example is a non-commutative version of the delta distribution : pick $x_{0} \in M$, and let $\pi$ be a unitary representation of $G$. Then

$$
\mathcal{U}: f \in C_{0}^{\infty}(M, G) \rightarrow \mathcal{U}(f)=\pi\left[f\left(x_{0}\right)\right]
$$

is a non-commutative distribution on $M$, located at $x_{0}$. The first non-located $G$-valued distribution was constructed in [14], [15] as follows. Let $\pi$ be an orthogonal representation of $G$ in the Hilbert space $\mathcal{K}$, and let $\beta \in Z^{1}(G, \mathcal{K})$ be a continuous $\mathcal{K}$-valued 1-cocycle (i.e. $\beta: G \rightarrow \mathcal{K}$ is such that $\left.\beta\left(g g^{\prime}\right)=\beta(g)+\pi(g) \cdot \beta\left(g^{\prime}\right)\right)$ for all $\left.g, g^{\prime} \in G\right)$. Let $\mu$ be a positive non-atomic Radon measure on $M$, and introduce the Hilbert integral :

$$
\widetilde{\mathcal{K}}=\int_{M}^{\oplus} d \mu(x) \mathcal{K}_{x}
$$

where $\mathcal{K}_{x} \cong \mathcal{K}, x \in M$.

One then has an action of $C_{0}^{\infty}(M, G)$ on $\widetilde{\mathcal{K}}$ :

$$
\tilde{\pi}(f)=\int_{M}^{\oplus} d \mu(x) \pi[f(x)] \in G L(\mathcal{K})
$$

and an associated one-cocycle :

$$
\widetilde{\beta}(f)=\int_{M} d \mu(x) \beta[f(x)] \in \widetilde{\mathcal{K}}
$$

Let now $\widetilde{\mathcal{H}}=S \overline{\mathcal{K}}_{\mathbf{C}}$ be the symmetric Hilbert space of $\overline{\mathcal{K}}_{\mathbf{C}}$, the complexification of the closure $\overline{\mathcal{K}}$ of $\widetilde{\mathcal{K}}$ w.r.t. the norm $\|\cdot\|=<\cdot, \cdot>^{\frac{1}{2}}$

$$
\widetilde{\mathcal{H}}=\sum^{\oplus} S_{n} \overline{\mathcal{K}}_{\mathbf{C}}=\sum^{\oplus} \overline{\mathcal{K}}_{\mathbf{C}}^{\oplus n}
$$


spanned by the coherent vectors

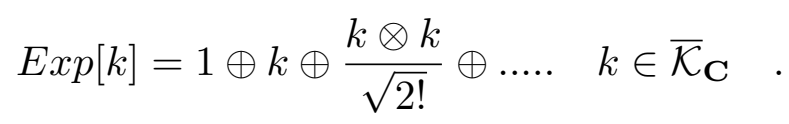

From $\widetilde{\pi}$ and $\widetilde{\beta}$ one can build a unitary (projective) representation of $C_{0}^{\infty}(M, G)$ on $\widetilde{\mathcal{H}}$, as follows :

$$
\mathcal{U}[f] \cdot \operatorname{Exp}[h]=e^{-\frac{1}{2}\|\widetilde{\beta}(f)\|^{2}} e^{-<\widetilde{\pi}(f) \cdot h, \widetilde{\beta}(f)>} \operatorname{Exp}[\widetilde{\pi}(f) \cdot h+\widetilde{\beta}(f)]
$$

the projective factor (or 2-cocycle) being given by

$$
\omega(f, g)=\operatorname{Im}<\widetilde{\beta}(f), \widetilde{\pi}(f) \cdot \widetilde{\beta}(g)>
$$

Moreover, this projective representation can be turned into a true representation if one can find some function $\gamma: G^{M} \rightarrow \mathbf{R}$ such that :

$$
\omega(f, g)=\gamma(f \cdot g)-\gamma(f)-\gamma(g)
$$

Then $\mathcal{U}_{\gamma}=e^{-i \gamma} \cdot \mathcal{U}$ is a true representation of $G$.

Remark : If one chooses to work with real Hilbert spaces only, then the 2-cocycle vanishes identically, and $\mathcal{U}=\mathcal{U}_{0}$ is a true representation.

The starting point of the above construction was a unitary representation $\pi$ of $G$, and a continuous 1-cocycle $\beta$. It has been shown in [14] and [21] that a necessary condition for $\mathcal{U}$ to be irreducible is that $\beta \notin B^{1}(G, \mathcal{K})$. However, the groups $H^{1}(G, \mathcal{K})$ are closely connected to the neighborhood of the trivial representation in the unitary dual $\widehat{G}$ of $G$.

$G$ is said to satisfy the $\Gamma$ property if the trivial representation is not isolated in $\widehat{G}$ [22], (we recall that the canonical topology in $\widehat{G}$ is not Hausdorff separated). In [23] and [21], it was shown that if $G$ has the $\Gamma$ property, then $H^{1}(G, \mathcal{H}) \neq\{0\}$ for some irreducible unitary representation $\pi: G \rightarrow \mathcal{U}(\mathcal{H})$.

Moreover, if the irreducible unitary representation $\pi: G \rightarrow \mathcal{U}(\mathcal{H})$ is such that $H^{1}\left(G, \mathcal{H} \neq\{0\}\right.$, then $\pi$ is non separated from the trivial representation $\mathbb{1}_{G}$ in $\widehat{G}$, [24] : $\left(\pi\right.$ is called in [24] an infinitely small representation of $G$ ). The neighborhood of $\mathbb{1}_{G}$ in $\widehat{G}$ is then an interesting object to study. Notice that in [15], the continuous tensor product representation is also realised as an inductive limit of finite tensor products fo "smaller 
and smaller" representations of $G$ (in that case in the supplementary series of $S L(2, \mathbf{R})$ ), converging to the trivial one.

\section{III - The jet groups $G_{m}^{k}$ and their representations :}

In the case of compact groups, all unitary irreducible representations are squareintegrable, and form a discrete series. The trivial representation is then isolated, and the previous construction fails. However, such a disease can be cured by the following trick : it is sufficient to find an embedding :

$$
j: G \hookrightarrow G^{\prime}
$$

into a bigger group $G^{\prime}$, such that $H^{\prime}\left(G^{1}, \mathcal{H}\right)$ is non trivial for some irreducible unitary representation in $\mathcal{H}$. Then one can build continuous tensor product representations of $G^{M}$, and use the $j$ embedding to get a representation of $G^{M}$. The irreducibility of such representations must be studied separately. In [18], and in [20], [25], the authors considered the first jet groups $G_{m}^{1}$ of $G$ to start with, mentioning the possibility of using higher order extensions. We consider here jet groups of order 1,2 and 3 .

\section{III - 1 Structure of jet groups :}

Let $G$ be a locally compact Lie group, and $M$ a Riemannian manifold of dimension $m$. With $G$ and $M$ are canonically associated the corresponding jet extensions $G_{m}^{k}$, defined as follows. Consider the current group $C_{0}^{k}(M, G)$, and the subgroup

$$
G_{x_{0}, k}^{M}=\left\{\varphi \in C_{0}^{k}(M, G), \varphi\left(x_{0}\right)=e,\left(D^{\alpha} \varphi\right)\left(x_{0}\right)=0 \quad \forall \alpha \in \mathbf{N}^{m}, 1 \leq|\alpha| \leq k\right\}
$$

for some $x_{0} \in M$. It follows from Leibniz's derivation rule that $G_{x_{0}, k}^{M}$ is a normal subgroup of the current group $C_{0}^{k}(M, G)$, so that the coset space

$$
G_{m}^{k}=C_{0}^{k}(M, G) / G_{x_{0}, k}^{M}
$$

inherits a group structure ${ }^{(1)}$. Let $[\varphi] \in G_{m}^{k}$ denote the equivalence class of $\varphi \in C_{0}^{k}(M, G)$. Then if $\varphi, \psi \in C_{0}^{k}(M, G)$ :

(1) It is easy to see that $G_{m}^{k}$ does not depend on $x_{0}$, which justifies our notation. 


$$
[\varphi][\psi]=[\varphi \cdot \psi]
$$

Moreover, if $\varphi \in C_{0}^{k}(M, G)$, and modulo a right-trivialization, $[\varphi]$ is in a bijective correspondence with

$$
[\varphi] \cong\left(\varphi\left(x_{0}\right),\left(d \varphi \cdot \varphi^{-1}\right)\left(x_{0}\right), \ldots\left[d^{k-1}\left(d \varphi \cdot \varphi^{-1}\right)\right]\left(x_{0}\right)\right)
$$

where $\left(d \varphi \cdot \varphi^{-1}\right)\left(x_{0}\right)$ denotes as usual the value obtained by right-translation by $\varphi^{-1}\left(x_{0}\right)$ of $d \varphi^{-1}\left(x_{0}\right) \in T_{\varphi^{-1}\left(x_{0}\right)} M$. To simplify the notations, we shall omit the $x_{0}$ - argument from now on.

To specify a bit more the structure of $G_{m}^{k}$, it is necessary to introduce some more ingredients.

Denote by $\mathcal{G}=$ Lie $(G)$ the Lie algebra of $G$, and let $A(n)$ denote the following abelian group :

$$
A(n)=\mathcal{G}^{n}=\mathcal{G} \times \mathcal{G} \times \ldots \times \mathcal{G} \quad(n \text { times }),
$$

the direct product of $n$ copies of $\mathcal{G}$, considered as an abelian group. On $A(n)$ one has an action of $G$, as the direct product of $n$ adjoint actions of $G$ on $\mathcal{G}$ :

$$
\operatorname{ad}(g) \cdot\left(x_{1}, \ldots x_{n}\right)=\left(\left(\operatorname{ad}(g) \cdot x_{1}, \ldots a d(g) \cdot x_{n}\right) \quad g \in \mathcal{G}\right.
$$

In what follows, we will need two composition laws, denoted by $[,]_{\otimes}$ and $[,]^{\otimes}$ respectively, and defined by :

$$
\begin{gathered}
{[,]_{\otimes}: x, y \in A(n) \rightarrow[x, y]_{\otimes} \in A\left(n^{2}\right)} \\
\left\{[x, y]_{\otimes}\right\}_{i, j}=\left[x_{i}, y_{j}\right]
\end{gathered}
$$

and

$$
[,]^{\otimes}:(x, y) \in A(n) \times A(n) \rightarrow[x, y]^{\otimes} \in A\left(n^{2}\right)
$$

by duality, using the (positive-definite) killing form :

$$
<[x, y]^{\otimes}, z>=<x,[y, z]_{\otimes}>\quad \forall z \in A\left(m^{2}\right)
$$


We will also make use of the following property, the proof of which follows from simple computations:

\section{Lemma 2 :}

Let $\varphi \in C_{0}^{k}(M, G)$, and $X \in A(m)$. Then

$$
d[\operatorname{ad}(\varphi) \cdot X]=\left[d \varphi \cdot \varphi^{-1}, \operatorname{ad}(\varphi) \cdot X\right]_{\otimes}+a d(\varphi) \cdot d X
$$

We are now in position to show :

\section{Proposition 3 :}

i)

$$
G_{m}^{k} \cong G \mid \times N_{m}^{k}
$$

where $N_{m}^{k}$ is topologically isomorphic to

$$
N_{m}^{k} \cong A(m) \times A\left(m^{2}\right) \times \ldots \times A\left(m^{k}\right)
$$

and the action of $G$ on $N_{m}^{k}$ is the adjoint action.

ii) $N_{m}^{1}$ is the abelian group $A(m)$.

iii) $N_{m}^{2}$ is a central extension of $A(m)$ by $A\left(m^{2}\right)$.

$$
\left(x_{1}, y_{1}\right)\left(x_{2}, y_{2}\right)=\left(x_{1}+x_{2}, y_{1}+y_{2}+\left[x_{1}, x_{2}\right]_{\otimes}\right) \quad\left(x_{1}, y_{1}\right)\left(x_{2}, y_{2}\right) \in N_{m}^{2}
$$

iv) $N_{m}^{3}$ is the semi-direct product of $A(m)$ by the (non-abelian) group $A\left(m^{2}\right) \times A\left(m^{3}\right)$

$$
\begin{aligned}
& N_{m}^{3} \cong A(m) \times\left[A\left(m^{2}\right) \times A\left(m^{3}\right)\right] \\
&\left(x_{1}, y_{1}, z_{1}\right)\left(x_{2}, y_{2}, z_{2}\right)=\left(x_{1}+x_{2}, y_{1}+y_{2}+\left[x_{1}, x_{2}\right]_{\otimes}, z_{1}+z_{2}+2\left[x_{1}, y_{2}\right]_{\otimes}\right. \\
&+\left[y_{1}, x_{2}\right]_{\otimes}+\left[x_{1},\left[x_{1}, x_{2}\right]_{\otimes}\right]_{\otimes} \\
&\left(x_{1}, y_{1}, z_{1}\right),\left(x_{2}, y_{2}, z_{2}\right) \in N_{m}^{3}
\end{aligned}
$$

Proof : The proof follows from explicit computations 
i) is a direct consequence of (III.5).

ii) Using (III.4) and (III.5), we have :

$$
\begin{aligned}
\left(\varphi, d \varphi \cdot \varphi^{-1}\right)\left(\psi, d \psi \cdot \psi^{-1}\right) & \left.=\left(\varphi \psi, d(\varphi \psi)(\varphi \psi)^{-1}\right)\right) \\
& =\left(\varphi \psi, d \varphi \cdot \varphi^{-1}+a d(\varphi) \cdot\left(d \psi \cdot \psi^{-1}\right)\right)
\end{aligned}
$$

iii) We restrict to the third argument of $\left(\varphi,\left(d \varphi \cdot \varphi^{-1}\right), d\left(d \varphi \cdot \varphi^{-1}\right)\right) \in N_{m}^{2}$, and use Lemma 2. Then

$$
\begin{aligned}
d\left[d \varphi \cdot \varphi^{-1}\right. & \left.+a d(\varphi) \cdot\left(d \psi \cdot \psi^{-1}\right)\right]=d\left(d \varphi \cdot \varphi^{-} 1\right) \\
& +\left[d \varphi \cdot \varphi^{-1}, a d(\varphi) \cdot\left(d \psi \cdot \psi^{-1}\right)\right]_{\otimes}+a d(\varphi) \cdot\left[d\left(d \psi \cdot \psi^{-1}\right)\right]
\end{aligned}
$$

which yields (III.15).

iv) We now focus on the fourth component of the product

$\left(\varphi, d \varphi \cdot \varphi^{-1}, d\left(d \varphi \cdot \varphi^{-1}\right), d^{2}\left(d \varphi \cdot \varphi^{-1}\right)\right) \cdot\left(\psi, d \psi \cdot \psi^{-1}, d\left(d \psi \cdot \psi^{-1}\right), d^{2}\left(d \psi \cdot \psi^{-1}\right)\right)$ that reads

$$
\begin{aligned}
d\left[d\left(d \varphi \cdot \varphi^{-1}\right)+\left[\left(d \varphi \cdot \varphi^{-1}\right), a d(\varphi) \cdot\left(d \psi \cdot \psi^{-1}\right)\right]_{\otimes}+a d(\varphi) \cdot d\left(d \psi \cdot \psi^{-1}\right)\right] \\
=d^{2}\left(d \varphi \cdot \varphi^{-1}\right)+\left[d\left(d \varphi \cdot \varphi^{-1}\right), a d(\varphi) \cdot\left(d \psi \cdot \psi^{-1}\right)\right]_{\otimes} \\
\quad+2\left[\left(d \varphi \cdot \varphi^{-1}\right), a d(\varphi) \cdot\left[d\left(d \psi \cdot \psi^{-1}\right)\right]\right]_{\otimes}+a d(\varphi) \cdot d^{2}\left(d \psi \cdot \psi^{-1}\right) \\
\quad+\left[\left(d \varphi \cdot \varphi^{-1}\right),\left[d \varphi \cdot \varphi^{-1}, a d(\varphi) \cdot\left(d \psi \cdot \psi^{-1}\right)\right]_{\otimes}\right]_{\otimes}
\end{aligned}
$$

and this proves (III.17) and completes the proof of the proposition.

In the next section, we will also use the following properties of $N_{m}^{3}$, that are direct consequences of proposition 3 :

\section{Lemma 4 :}

i) Let $(x, y, z) \in N_{m}^{3}$. Then

$$
\begin{aligned}
(x, y, z)^{-1}= & \left(-x,-y+[x, x]_{\otimes},-z+2[x, y]_{\otimes}+[y, x]_{\otimes}\right. \\
& \left.-\left[x,[x, x]_{\otimes}\right]_{\otimes}\right)
\end{aligned}
$$

ii) $A\left(m^{2}\right) \times A\left(m^{3}\right)$ is an abelian normal subgroup of $N_{m}^{3}$ : if $(b, c) \in A\left(m^{2}\right) \times A\left(m^{3}\right)$ : 


$$
a d(x, y, z) \cdot(0, b, c)=\left(0, b, c+2[x, b]_{\otimes}-[b, x]_{\otimes}\right)
$$

$\forall(x, y, z) \in N_{m}^{3}$

\section{III - 2 Representation theory}

We will now make use of the assumption that $G$ is compact simple. We discuss in this section the representation theory of the previously considered jet groups. More precisely, we will focus on the neighborhood of the trivial representation in their unitary dual $\widehat{G_{m}^{k}}$. In all cases, we will use Mackey's induction theory (see [26] for a review).

\section{a) First order group :}

The case of the first order Leibniz group is fairly simple, and the result is already well known (see e.g. [26]). We sketch the construction here. Let

$$
\chi_{T}=e^{i<T, \cdot>}
$$

be a multiplicative character of $A(m)$, and let $\mathcal{O}_{T}$ be the $G$-orbit through $\chi_{T}$ (here $T \in \mathcal{G}^{\star} \oplus \ldots \oplus \mathcal{G}^{\star}$, where $\mathcal{G}^{*}$ is the dual of $\left.\mathcal{G}\right)$. Let $G_{T} \subset G$ be the stability group of $\chi_{T}$ :

$$
G_{T}=\left\{g \in G_{m}^{1}, a d^{\star}(g) \cdot \chi_{T}=\chi_{T}\right\}
$$

As usual, $A(m)$ is a normal subgroup of $G_{T}$, and

$$
G_{T}=H_{T} \times A(m)
$$

( $\times$ standing for semidirect product) where

$$
H_{T}=G_{T} / A(m)
$$

Let

$$
T=\left(t_{1}, \ldots t_{m}\right) \in A(m)^{\star}=\mathcal{G}^{\star} \oplus \ldots \oplus \mathcal{G}^{\star}
$$

Since we will focus on the neighborhood of the trivial representation, we can restrict ourselves to the case of regular $t_{i}$ 's. Then $H_{T}$ is a torus of $G$, of dimension say $n$. It is easy to see that $\chi_{T}$ can be extended to a character of $G_{T}$, denoted by $\chi_{T}^{e}$ : 


$$
\chi_{T}^{e}(h, x)=\chi_{T}(x) \quad(h, x) \in G_{T} .
$$

Let $\chi_{\alpha}: H_{T} \rightarrow \mathbf{C}$

$$
\chi_{\alpha}(\xi)=\xi^{\alpha} \quad \xi \in H_{T}
$$

be an arbitrary character of the compact abelian group $H_{T}$ and let $\chi_{\alpha}^{e}$ be its extension to $G_{T}$. Let finally

$$
\chi=\chi_{\alpha}^{e} \otimes \chi_{T}^{e}
$$

denote the Kronecker product of $\chi_{\alpha}^{e}$ and $\chi_{T}^{e}$. Then, since $N_{m}^{1}$ is of type I, the correspondence

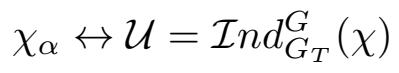

is a one-to-one correspondence between

- The characters of $H_{T}$

- The unitary irreducible representations of $G_{m}^{1}$ whose quasi-orbits are concentrated on $\mathcal{O}_{T}$.

Let us now study the limit $T \rightarrow 0$ (i.e. $t_{i} \rightarrow 0, i=1, \ldots m$ ). $\mathcal{U}$ acts on the Hilbert space

$$
\mathcal{H} \cong L^{2}\left(G_{T} \backslash^{G_{m}^{1}}\right) \cong L^{2}\left(H_{T} \backslash^{G}\right)
$$

Let $u, v \in \mathcal{H}$. A simple calculation shows that

$$
<u, \mathcal{U}(g, x) \cdot v>\underset{T \rightarrow 0}{\longrightarrow} u(e) v(e)
$$

so that $\mathcal{U} \rightarrow \mathbb{1}$ weakly. We have then exhibited a sequence of non-trivial irreducible unitary representations converging to the trivial one. Thus the trivial representation is not isolated in $\widehat{G_{m}^{1}}$.

Remark : Set directly $T=0$; then $\chi_{T}$ extends to the trivial representation of $G_{m}^{1}$, and then any representation of $G_{m}^{1}$ that is trivial on $N_{m}^{1}$ is an extension $\pi^{e}$ of an irreducible unitary representation $\pi$ of $G$ on $\mathcal{H}$. Let $\beta$ be a continuous 1-cocycle associated with $\pi^{e}$. It then follows from the cocycle property that 


$$
(e, x)=\pi(g) \cdot \beta\left(e, a d(g)^{-1} \cdot x\right) \quad \forall(g, x) \in G_{m}^{1}
$$

This implies that the linear operator $U: x \in g \rightarrow U \cdot x=\beta(e, x) \in \mathcal{H}$ intertwines $\pi$ and the adjoint representation, if $\beta$ is non-trivial. Then the non-triviality of $\beta$ implies that $\pi$ is unitarily equivalent to the adjoint representation. An example of a non-trivial 1 -cocycle is well known : the so-called Maurer-Cartan cocycle $\beta(g, x)=x$.

\section{b) Second order group :}

As stressed in Proposition $3, N_{m}^{2}$ is a central extension of $A(m)$ by $A\left(m^{2}\right)$. We shall now study the representations of $N_{m}^{2}$. Let

$$
\chi_{T}=e^{i<T, \cdot>}
$$

be a multiplicative character of $A\left(m^{2}\right)$. Here $T \in A\left(m^{2}\right)^{\star}$.

Denote by $N_{T}=\left\{n \in N_{m}^{2}, A d^{\star}(n) \cdot T=0\right\}$ the stability group $T . N_{T}$ is a normal subgroup. Set

$$
H_{T}=N_{T} / A\left(m^{2}\right)
$$

We set $H_{0}=A(m) / H_{T}$. Let $B_{T}$ be the skew 2-form on $A(m)$, defined by

$$
B_{T}\left(x, x^{\prime}\right)=<T,\left[x, x^{\prime}\right]_{\otimes}>\quad x, x^{\prime} \in A(m)
$$

$B_{T}$ is clearly non-degenerate on $H_{0}$. It is then possible to find a maximal isotropic $H_{0}$-subgroup, denoted by $H_{2}$ :

$$
H_{0}=H_{1} \times H_{2}
$$

Let $\chi_{\alpha}=e^{i<\alpha, \cdot>}$ and $\chi_{\beta}=e^{i<\beta, \cdot>}$ be two characters of $H_{2}$ and $H_{T}$ respectively and let

$$
\chi_{\alpha \beta}=\chi_{\alpha}^{e} \otimes \chi_{\beta}^{e}
$$

where $\chi_{\alpha}^{e}$ and $\chi_{\beta}^{e}$ are the extensions of $\chi_{\alpha}$ and $\chi_{\beta}$ to $H_{0}$. Finally, if $\chi_{\alpha \beta}^{e}$ and $\chi_{T}^{e}$ are the extensions of $\chi_{\alpha \beta}$ and $\chi_{T}$ to $N_{T}$, the correspondence

$$
\chi_{\alpha \beta} \rightarrow \chi=\chi_{\alpha \beta}^{e} \otimes \chi_{T}^{e}
$$


is a one-to-one correspondence between the characters of $H_{2}$ and the characters of $N_{T}$ whose restriction to $A\left(m^{2}\right)$ is $\chi_{T}$.

To proceed further, let us induce from $N_{T}$ to $N_{m}^{2}$. Let

$$
\chi\left(h_{2}+z, y\right)=e^{i<\alpha, h_{2}>} e^{i<\beta, z>} e^{i<T, y>}, \quad h_{2} \in H_{2}, z \in H_{T}, y \in A\left(m^{2}\right)
$$

Let $H=H_{2} \times H_{T} \times A\left(m^{2}\right)$

$$
\begin{aligned}
\mathcal{H}=\left\{f: N_{m}^{2} \rightarrow \mathbf{C}, \text { measurable, } f \in L^{2}\right. & \left(H \backslash^{N_{m}^{2}}, \mu\right), \\
& \left.f(h n)=\chi(h) f(n) \quad \forall h \in H, n \in N_{m}^{2}\right\}
\end{aligned}
$$

for some quasi-invariant measure $\mu$ on ${ }_{H} \backslash^{N_{m}^{2}}$, and let $\mathcal{U}=\operatorname{Ind} d_{H}^{N_{m}^{2}}(\chi)$. Then

$$
\begin{aligned}
& {\left[\mathcal{U}\left(h_{1}, h_{2}, z, y\right) \cdot f\right](x, 0,0,0)=e^{i<\alpha, h_{2}>} e^{i<\beta, z>}} \\
& \quad e^{i<T, y+z\left[x, h_{2}\right]_{\otimes}+\left[x, h_{1}\right]_{\otimes}+\left[h_{1}, h_{2}\right]_{\otimes}>} f\left(x+h_{1}, 0,0,0\right)
\end{aligned}
$$

Let us now look for representations of $G_{m}^{2}$. Starting from the previous representation $\mathcal{U}$, one looks for its stability $G_{m}^{2}$-subgroup :

$$
G_{\mathcal{U}}=\left\{(g, x, y) \in G_{m}^{2}, \quad \mathcal{U}^{(g, x, y)} \cong \mathcal{U}\right\}
$$

where

$$
\mathcal{U}^{(g, x, y)}(n)=\mathcal{U}[a d(g, x, y) \cdot n] \quad n \in N_{m}^{2},(g, x, y) \in G_{m}^{2}
$$

Then, with $\cong$ denoting isomorphism:

$$
G_{\mathcal{U}} \cong\left\{g \in G, a d^{\star}(g) \cdot \alpha=\alpha, a d^{\star}(g) \cdot \beta=\beta, a d^{\star}(g) \cdot T=T\right\} \mid \times N_{m}^{2}
$$

and if $\alpha, \beta, T$ are regular elements of $A\left(m^{2}\right), G_{\mathcal{U}}$ is a torus of $G$, times $N_{m}^{2}$.

In general, $\mathcal{U}$ does not extend to a representation of $G_{\mathcal{U}}$. However, by general results [26], there exists a (unique up to equivalence) 2-cocycle $\sigma$ of $G_{\mathcal{U}} / N_{m}^{2}$ and a 
$\sigma$-representation $\mathcal{U}^{e}$ of $G_{\mathcal{U}}$ that extends $\mathcal{U}$ to $G_{\mathcal{U}}$. Let $\mathcal{V}$ be a $\frac{1}{\sigma}$-representation of $G_{\mathcal{U}} / N_{m}^{2}$, let $\mathcal{V}^{e}$ the representation of $G_{\mathcal{U}}$ defined by

$$
\mathcal{V}^{e}(n g)=\mathcal{V}^{e}(g) \quad \forall n \in N_{m}^{2}, g \in G
$$

and define

$$
\Gamma=\mathcal{V}^{e} \otimes \mathcal{U}^{e}
$$

$\Gamma$ is a unitary representation of $G_{\mathcal{U}}$ on some Hilbert space $\mathcal{K}$, and all the representations of $G_{m}^{2}$ are obtained as

$$
\Pi=\mathcal{I} n d_{G_{\mathcal{U}}}^{G_{m}^{2}}(\Gamma)
$$

The representation space of $\Pi$ is

$$
\begin{aligned}
& \mathcal{H}_{\Pi}=\left\{f: G_{m}^{2} \rightarrow \mathcal{K}\right. \text {, measurable; } \\
& \left.\qquad f \in L^{2}\left(G_{\mathcal{U}} \backslash G^{2}\right) ; f(h g)=\Gamma(h) \cdot f(g) \forall h \in G_{\mathcal{U}}\right\}
\end{aligned}
$$

and $\Pi$ acts on $f \in \mathcal{H}_{\Pi}$ as

$$
[\Pi(g, x, y) \cdot f]\left(h, x^{\prime}, y^{\prime}\right)=f\left(h, x^{\prime}, y^{\prime}\right)(g, x, y)
$$

Setting $\alpha=\beta=0$, and letting $T \rightarrow 0$, one can see that such a sequence of irreducible unitary representation converges to the extension of $\mathcal{U}$ to $G_{m}^{2}$, which is nontrivial.

Thus the trivial representation is an isolated point in $\widehat{G_{m}^{2}}$, and

$$
H^{1}\left(G_{m}^{2}, \mathcal{H}\right)=\{0\}
$$

for any irreducible unitary $G_{m}^{2}$-module $\mathcal{H}$.

Remark : J. Pichaud [27] has proved that if $H$ is a closed cocompact subgroup of a locally compact locally connected separable group $G$, then

$$
H^{1}\left(G, \mathcal{H}^{\prime}\right) \cong H^{1}(H, \mathcal{H})
$$


for any separable irreducible unitary $H$-module $\mathcal{H}$, and with $\mathcal{H}^{\prime}$ the $G$-module induced from $\mathcal{H}$ in the sense of Mackey. In the case of $G_{m}^{2}$, it is easy to see from (III.41) - (III.46) that the trivial representation is isolated in $\widehat{G_{\mathcal{U}}}$, which in turn implies (III.49).

\section{c) Third order group :}

Since $N_{m}^{3}$ is connected and nilpotent one knows that $H^{1}\left(N_{m}^{3}, \mathcal{H}\right)=\{0\}$ for any irreducible unitary $N_{m}^{3}$-module $\mathcal{H}$. However, it is not possible to use directly such a result for the representations of $G_{m}^{3}$.

We first look at the representations of $N_{m}^{3}$. It follows from section III.1 that

$$
N_{m}^{3}=A(m) \times A
$$

where $A=A\left(m^{2}\right) \times A\left(m^{3}\right)$ is an abelian group.

Let $T=\left(\tau_{y}, \tau_{z}\right) \in A\left(m^{2}\right)^{\star} \oplus A\left(m^{3}\right)^{\star}$, and let

$$
\chi_{T}(y, z)=e^{i<\tau_{y}, y>} e^{i<\tau_{z}, z>},(y, z) \in A
$$

be the corresponding character of $A$. Let $n=(x, y, z) \in N_{m}^{3}$, and set

$$
\begin{aligned}
\chi_{T}^{n}(u, v) & =\chi_{T}(\operatorname{ad}(x, y, z) \cdot(u, v)) \\
& =\chi_{T}\left(u, v+3[x, u]_{\otimes}\right)
\end{aligned}
$$

Then $\chi_{T}^{n} \cong \chi_{T}$ if and only if $\left[x, \tau_{z}\right]_{\otimes}=0$. Let $N_{T}=\left\{n \in N_{m}^{3}, \chi_{T}^{n} \cong \chi_{T}\right\}$, and assume that all the components of $\tau_{z}$ are regular elements of $\mathcal{G}$. Then $N_{T} / A$ is an intersection of Cartan subalgebras of $\mathcal{G}$, and is thus abelian. Extend $\chi_{T}$ to $N_{T}$ by

$$
\chi_{T}^{e}(x, y, z)=\chi_{T}(y, z) \quad(x, y, z) \in N_{T}
$$

A simple calculation shows that $\chi_{T}^{e}$ is indeed a representation of $N_{T}$. Now let $\chi_{\alpha}$ be a character of $N_{T}(A), \chi_{\alpha}^{e}$ its extension to $N_{T}$, and set :

$$
\chi=\chi_{\alpha} \otimes \chi_{T}
$$

Let $\mathcal{U}=\mathcal{I} n d_{N_{T}}^{N_{m}^{3}}(\chi) \cdot \mathcal{U}$ acts on 


$$
\begin{gathered}
\mathcal{H}=\left\{f: N_{m}^{3} \rightarrow \mathbf{C}, \text { measurable, } \int_{N_{T} \backslash N_{m}^{3}}|f|^{2} d \mu<\infty,\right. \\
\left.f(h n)=\chi(h) f(n) \quad \forall h \in N_{T}\right\}
\end{gathered}
$$

as :

$$
\begin{aligned}
& {[\mathcal{U}(n+\xi, y, z) \cdot f](u)=\chi_{\alpha}(\xi) e^{i<\tau_{y}, y+2[u, \xi]_{\otimes}+[u, n]_{\otimes}-[\xi, n]_{\otimes}>}} \\
& \quad e^{i<\tau_{z}, z+3[u, y]_{\otimes}+[n, y]_{\otimes}+2\left[u[u, n+\xi]_{\otimes}\right]_{\otimes}+\left[n,[u, n+\xi]_{\otimes}\right]_{\otimes}-\left[\xi,[\xi, u+n]_{\otimes}\right]_{\otimes}>} \\
& \quad f(u+n)
\end{aligned}
$$

Let $G_{T}=H_{T} \times A$ be the stability group of $\mathcal{U}$. Then $H_{T}$ is a torus in $G$, and $\mathcal{U}$ extends to a $\sigma$-representation $\mathcal{U}^{e}$ of $G_{T}$ (for some $\sigma \in H^{2}\left(H_{T}, S^{1}\right)$ ). Let $\tau \in H^{2}\left(H_{T}, S^{1}\right)$, cohomologous to $\sigma^{-1}$. Then if $\mathcal{V}$ is any irreducible $\tau$-representation of $H_{T}$ and $\mathcal{V}^{e}$ its extension to $G_{T}$, the correspondence

$$
\mathcal{V} \leftrightarrow \Pi=\mathcal{I}_{n d_{G_{T}}^{G}}^{G}\left(\mathcal{V}^{e} \otimes \mathcal{U}^{e}\right)
$$

is a one-to-one correspondence $[26]$.

The same arguments as in section III.2.a permit to exhibit generalized sequences of nontrivial unitary irreducible representations that converge weakly to the trivial one. Nevertheless, it is not possible to deduce the triviality or non-triviality of the $H^{1}\left(G_{m}^{3}, \mathcal{H}\right)$ cohomology groups from such a result alone, and we have to leave open this problem for the time being.

\section{d) Summary :}

The results of the current section can be summarized as follows :

\section{Theorem 5 :}

Let $G_{m}^{k}$ be the Leibniz extension of order $m$ and degree $k$ of a compact simple group $G$.

i) There exist irreducible unitary $G_{m}^{1}$-modules $\mathcal{H}$ such that $H^{1}\left(G_{m}^{1}, \mathcal{H}\right)$ is non-trivial.

ii) $H^{1}\left(G_{m}^{2}, \mathcal{H}\right)$ is trivial for any irreducible unitary $G_{m}^{2}$-module $\mathcal{H}$.

iii) The trivial representation is not isolated in $\widehat{G_{m}^{3}}$. 


\section{IV - Conclusion}

We have investigated in this paper the irreducible unitary representations of the three first Leibniz extensions $G_{m}^{k}$ of a compact simple Lie group $G$, focusing on the neighborhood of the trivial representation.

The motivation was the generalization of the energy representation of $\mathcal{D}(M, G)$ to such contexts. By known results, such a generalization is only possible when there exists an irreducible unitary representation $\pi: G_{m}^{k} \rightarrow \mathcal{U}(\mathcal{H})$ such that $H^{1}\left(G_{m}^{k}, \mathcal{H}\right)$ is nontrivial. Moreover the continuous cohomology groups $H^{1}\left(G_{m}^{k}, \mathcal{H}\right)$ are closely related to the structure of $\widehat{G_{m}^{k}}$, and more precisely to that of the neighborhood of the trivial representation.

We have shown that the trivial representation is isolated in $\widehat{G_{m}^{2}}$, while it is not isolated in $\widehat{G_{m}^{1}}$ and $\widehat{G_{m}^{3}}$. This implies that all the unitary representation valued cohomology groups of $G_{m}^{2}$ are trivial, and that there does not exist a second-order generalization of the energy representation. The order one case was already studied before, and leads to the standard energy representation and some generalizations [7]. In the case of third order Leibniz group, our result, that the trivial representation is not isolated in $\widehat{G_{m}^{3}}$, does not permit by itself to deduce the triviality or nontriviality of the $H^{1}$ groups. This shows the limits of the approach we have taken. The trival representation can be non-isolated in $\widehat{G_{m}^{k}}$, without being able to deduce relevant informations about triviality or non-triviality of the corresponding $H^{1}$ groups. Another limit of our approach is that the structure of the nilpotent groups is too complex to allow explicit computations at any order. $N_{m}^{1}$ is quite simple, $N_{m}^{2}$ a bit more complex, and $N_{m}^{3}$ much more difficult.

It would be much better to try to compute directly the $H^{1}$ groups, using spectral sequences, but at present this has not been done.

\section{Acknowledgements :}

We are greatly indebted to Professor Daniel Testard for his constructive criticism and most helpful discussions. We are also grateful to Professors Helge Holden and Jean Marion for interesting and stimulating discussions.

This research has been facilitated by several common stays in Oslo, Marseille and Bochum, and we acknowledge partial financial support by the Institute of Mathematics of Oslo University, the Norwegian Science Foundation (NAVF), the Centre de Physique Théorique, CNRS, and the Université d'Aix-Marseille II, as well as the Alexander Von Humboldt - Stiftung and the research project Procope. 


\section{References}

$[1]$

$[3]$

$[4]$

$[5]$

$[6]$

$[7]$

[8]

$[9]$

$[10]$
V.G. Kac : Infinite-dimensional Lie algebras (third edition), Cambridge University Press (1991).

H.P. Jakobsen, V.G. Kac : A new class of unitarizable highest weight representations of infinite-dimensional Lie algebras, Lect. Notes in Phys. 226 (1985) pp. 1-20.

B. Torrésani : Representations Représentations projectives des groupes de transformations de jauge locales, Dissertation, Marseille (1986).

B. Torrésani : Unitary positive-energy representations of gauge groups, Lett. Math. Phys. 13 (1987), p.7.

B. Torrésani : Unitary highest weight representations of gauge groups, Preprint CPT-90/P.2361, in "Ideas and Methods in Mathematics and Physics", Oslo, October 1988, Albeverio, Lindstrm Ed. (1992), Cambridge University Press

R. Høegh-Krohn, B. Torrésani : Classification and construction of quasisimple Lie algebras, J. Funct. An. 89 (1990) pp. 106-136.

S. Albeverio, R. Høegh-Krohn, J. Marion, D. Testard, B. Torrésani : Non-commutative distributions, M. Dekker Publ. Comp., to appear.

H. Araki: Factorizable representations of current algebras, Publ. R.I.M.S. A5 (1970), pp. 361-422.

I.M. Gelfand, M.I. Graev : Représentations of quaternion groups over locally compact and functional fields, Funk. Anal. Pril. 2 (1968) pp. 20-35.

A. Guichardet : Symmetric Hilbert spaces and related topics, Lect. Notes in Math. 261 (1972) Springer.

S. Albeverio, R. Høegh-Krohn, D. Testard, A.M. Vershik : Factorial representations of path groups, J. Funct. An. 41 (1981) pp. 115-131.

H. Bosek, G. Czikowski, K.P. Rudolph, Analysis on topological groupsGeneral Lie theory, Teubner, Leipzig (1980). 
[13]

A. Ju. Daletzkij, Integrability of representations of nuclear Lie algebras of smooth currents (in Russian), in "Spectral theory of operators and infinite-dimensional analysis", Ak. Nauk. Ukrain. SSR (1984) pp. 77-91.

A.M. Vershik, I.M. Gelfand, M.I. Graev : Representations of the group $\mathrm{SL}(2, \mathrm{R})$ where $\mathrm{R}$ is a ring of functions, Russ. Math. Surv. 28 (1973) pp.83.

R.F. Streater : Canonical commutation relations, Continuous tensor products and infinitely divisible group representations, Rend. Sci. Inst. Fis. E. Fermi 11 (1969) pp. 247-263.

K.R. Parthasarathy, K. Schmidt: Factorizable representations of current groups and the Araki-Woods embedding theorem, Acta. Math. 128 (1972) pp. 53-71.

K.R. Parthasarathy, K. Schmidt: A new method for constructing factorizable representations of current groups and algebras, Comm. Math. Phys. 50 (1976) pp. 167-175.

R. Ismagilov : On unitary representations of the group $C_{0}^{\infty}(X, G)$, $G=S U(2)$, Math. U.S.S.R. Sb. 29 (1976) pp. 117-131.

S. Albeverio, R. Høegh-Krohn, : The energy representation of Sobolev - Lie groups, Comp. Math. 36 (1977) pp. 37-52.

I.M. Gelfand, M.I. Graev, A.M. Vershik : Representations of the group of functions taking values in a compact Lie group, Comp. Math. 35 (1977) pp. 299-334 ; Remarks on the representation of the group of functions with values in a compact Lie group, Comp. Math. 42 (1981) pp. 217-243.

P. Delorme : 1-Cohomologie des représentations unitaires des groupes de Lie semi-simples et résolubles. Produits tensoriels continus de représentations, Bull. Soc. Math. France 105 (1977) pp. 281-336.

D.A. Kazhdan: Funk. Anal. Appl. (in Russian) 1 (1967) p.63.

S.P. Wang, Proc. Am. Math. Soc. 42 (1974) p. 621. 
A.M. Vershik, S.I. Karpushev, Cohomology of groups in unitary representations, neighborhood of the unity and conditionally positive definite functions : Math. USSR. Sb. 47 (1973) pp.513-526. S. Albeverio, R. Høegh-Krohn, D. Testard : Irreducibility and reducibility for the energy representation of the group of mappings of a Riemannian manifold into a compact semisimple Lie group, J. Funct. An. 41 (1981) pp. 378-396. G.W. Mackey : Unitary representations of group extensions I, Acta. Math. 56 (1958) pp. 265-311.

J. Pichaud : 1-cohomologie de représentations induites, J. Math. pures et appl. 56 (1977) pp. 339-366. 